REV EXP MED

$2019 ; 5(1)$

\title{
Seroprevalencia de brucelosis en trabajadores de mataderos en la región de Lambayeque, Perú
}

\author{
Cinthya Y. Santa Cruz - López ${ }^{1 a}$, Ana M. Vásquez- Del Castillo ${ }^{1 b}$
}

\section{RESUMEN}

Objetivos: Determinar la seroprevalencia de brucelosis e identificar los factores predisponentes en trabajadores de mataderos de la región Lambayeque, durante los meses de Junio 2014 a Febrero del 2015. Materiales y métodos: Se realizó un muestreo aleatorio, obteniendo por venopunción 131 muestras sanguíneas de trabajadores de 27 mataderos municipales de la región Lambayeque. Se utilizaron las pruebas de Rosa de Bengala y Antígeno de Huddlenson para determinar la presencia de anticuerpos anti-Brucella sp. Para los factores predisponentes, se aplicó una encuesta validada por la Dirección General de Epidemiología. Resultados: La seroprevalencia de brucelosis fue del 10,7\%. Además se encontró que existe relación entre el consumo de sangre y la presencia de anticuerpos anti- Brucella sp. en los matarifes. El $83,5 \%$ de los mataderos no presentó condiciones higiénicas adecuadas y el $26,7 \%$ de los trabajadores evaluados no utilizaba medidas de protección durante las labores de matanza (uso de guantes, mandil, botas, mascarillas). Conclusiones: La seroprevalencia de brucelosis en los trabajadores de mataderos fue baja y el factor predisponente de mayor importancia para la transmisión de brucelosis fue el consumo de productos de animales infectados con Brucella sp. a saber sangre de los animales sacrificados.

Palabras clave: Estudios Seroepidemiológicos, Brucelosis, Mataderos (Fuente: DeCS-BIREME).

\section{Seroprevalence of brucellosis in workers of abattoirs in the region of Lambayeque, Peru}

\section{ABSTRACT}

Objectives: To determine brucellosis seroprevalence and to identify the predisposing factors in slaughterhouses' workers of Lambayeque region, during June 2014 to February 2015. Materials and methods: A random sampling was carried out. 131 blood samples from workers of 27 municipal slaughterhouses of Lambayeque region were obtained by venipuncture. Bengal Rose and Huddlenson antigen tests were employed to determine anti-Brucella sp antibodies' presence. For predisposing factors, a survey validated by the Epidemiology General Directorate was applied. Results: Brucellosis seroprevalence was of $10.7 \%$. Association between blood consumption and anti-Brucella sp antibodies' presence at slaughterhouses was also found. $83.5 \%$ of the slaughterhouses didn't hold adequate hygienic conditions and $26.7 \%$ of the evaluated workers didn't take protection measures during the slaughter (such as gloves, aprons, boots and masks). Conclusions: Brucellosis seroprevalance at slaughtehouses' workers was low and the most important predisposing factor for brucellosis transmission was the consumption of products from Brucella sp infected animals, specifically blood consumption from said slaughtered animals.

Keywords: Seroepidemiologic Studies, Brucellosis, Abattoirs (Fuente: MeSH-NLM).

\footnotetext{
${ }^{1}$ Facultad de Ciencias Biológicas, Universidad Nacional Pedro Ruiz Gallo, Lambayeque, Perú.

a Bióloga, maestra en microbiología clínica.

b Bióloga, doctora en microbiología. 


\section{INTRODUCCIÓN}

La brucelosis es una zoonosis con grandes repercusiones en la salud pública, generando un importante impacto social y económico, porque ocasiona enormes pérdidas anuales a la industria pecuaria, las que ascienden a US $\$ 600$ millones por brucelosis bovina en América Latina ${ }^{(1,2)}$. Además representa un verdadero riesgo ocupacional para los matarifes, carniceros, ganaderos, veterinarios y personal de laboratorio ${ }^{(3)}$.

Se estima que cada año se presentan 500 mil nuevos casos de brucelosis humana, debido a la alta prevalencia en los países en vías de desarrollo ${ }^{(2)}$. En el año 2004, en Perú se registraron 1116 casos de brucelosis humana (4); mientras que en el 2008 se notificaron 837 casos principalmente en las ciudades de Lima y Callao, reportándose la presencia de B. suis, B. abortus y B. canis. Recientemente en el año 2014 se encontraron dos casos de brucelosis humana en Lambayeque, sin embargo aún son muchos los casos por notificar ${ }^{(5)}$.

Lambayeque es una zona ganadera donde los caprinos, vacunos y porcinos son los principales animales explotados; y la brucelosis ha sido diagnosticada serológicamente en todos ellos ${ }^{(6)}$; constituyendo la población ganadera un reservorio de la bacteria y factor latente que predispone a la población humana a la infección por Brucella sp. Además, los pobladores lambayecanos se dedican a la producción de derivados lácteos, tales como cuajadas y quesos a partir de leche extraída de ganado caprino o bovino respectivamente; dichos productos elaborados sin control sanitario actúan también como vehículos de infección ${ }^{(7)}$.

Se considera que la fuente de infección más peligrosa son los productos del aborto, ya que contienen una elevada carga bacteriana; la que con gran facilidad puede transmitirse a través de la conjuntiva, piel con soluciones de continuidad o reblandecida, del sujeto sin protección ${ }^{(8)}$.

Se ha estimado que entre $10{ }^{(11)}$ y $10^{(14)}$ brucelas pueden ser eliminadas al medio ambiente durante un parto o aborto ${ }^{(9)}$; igual magnitud de riesgo de contaminación, presentan las vísceras, sangre y excretas de animales enfermos, por ello representan la forma de infección más común para veterinarios, ordeñadores, caballerangos, trabajadores de rastro, matadores ${ }^{(8)}$.

Otra vía de infección, es la inhalación de material contaminado desecado, como excretas, pelo y polvo de los corrales o bien por los aerosoles de bacterias vivas; en este caso la vía de entrada del germen es la respiratoria y ello se relaciona directamente con la ocupación del individuo ${ }^{(10,11)}$.

La brucelosis produce diversas manifestaciones clínicas, sin embargo en varias ocasiones se confunden los signos y síntomas con otros padecimientos o pasan desapercibidos ${ }^{(12)}$. Por ello se requieren estudios específicos de laboratorio para su diagnóstico, pero los estudios clínicos para aislar esta bacteria son escasos, costosos y poco oportunos, ya que tardan mucho tiempo y la mayoría de veces es necesario repetir la siembra porque no hay crecimiento bacteriano, lo que podría deberse al amplio uso de los antibióticos en los estados febriles de la enfermedad con anterioridad al diagnóstico, de tal manera que el indicador más cercano para conocer la real magnitud del problema es la utilización de pruebas serológicas ${ }^{(13)}$.

La prueba de Rosa Bengala es la técnica serológica más empleada para la evaluación de brucelosis que detecta anticuerpos aglutinantes empleando células de Brucella inactivadas, teñidas con Rosa de Bengala. El pH ácido contribuye a la especificidad de la prueba. La sensibilidad de la reacción es del $98 \%$ con una especificidad del $99 \%$. La prueba de Huddleson también es una reacción de aglutinación rápida, con una sensibilidad del $96 \%$ y especificidad de $99 \%$, presenta como antígeno una suspensión de B. abortus del $3 \%$ de gérmenes en fenol, verde brillante y cristal violeta y detecta anticuerpos tipo $\lg M, \lg G$ e $\lg \mathrm{A}^{(14)}$.

Por lo expuesto con anterioridad y considerando que no se conocen datos actuales sobre la brucelosis humana en la región de Lambayeque se plantearon como objetivos determinar la seroprevalencia e identificar los factores predisponentes de brucelosis en trabajadores de mataderos en la región de Lambayeque, durante los meses de Junio 2014 a Febrero del 2015.

\section{MATERIAL Y MÉTODOS}

\section{Tipo de estudio y diseño de la investigación}

La investigación fue tipo observacional, transversal y de temporalidad prospectiva. Se utilizó un diseño de prevalencia.

\section{Población y muestra}

La población estuvo constituida por los trabajadores de los 27 mataderos municipales pertenecientes a la región Lambayeque. El tamaño de la muestra fue calculado teniendo en consideración la fórmula estadística de proporciones y estuvo constituida por 131 trabajadores de ambos géneros, seleccionados al azar, durante los meses de Junio 2014 a Febrero 2015.

Para calcular el número trabajadores por matadero que fueron evaluados, se realizó un reparto proporcional de la muestra según método de prorrateo ${ }^{(15)}$, teniendo en cuenta el número de trabajadores de cada matadero. Se excluyeron a los trabajadores de mataderos no registrados (clandestinos) de la región Lambayeque.

\section{Técnicas e instrumentos de recolección de datos}

\section{Detección de la brucelosis}

La obtención de la sangre total, se realizó de acuerdo a lo 
establecido por el Ministerio de Salud del Perú ${ }^{(16)}$. La seroprevalencia de brucelosis se determinó utilizando el antígeno de Rosa de Bengala y Huddleson, para lo cual se llevó los reactivos y las muestras a temperatura ambiente, luego se resuspendió el antígeno, se aspiró y vació varias veces el cuentagotas para asegurar su homogeneidad.

Posteriormente se depositó una gota $(50 \mu \mathrm{L})$ de suero en un portaobjetos y se le colocó, próximo a la muestra a analizar, una gota de Rosa de Bengala (14).Se efectuó la mezcla con ayuda de un palillo desechable (mondadientes), extendiéndola de forma que cubra por completo la superficie. Se empleó palillos distintos para cada mezcla.

Por último, se movió el portaobjetos a mano o con agitador rotatorio durante cuatro minutos a $100 \mathrm{rpm}$ y de inmediato con la ayuda del microscopio, se observó la aparición de cualquier signo de aglutinación. Similar procedimiento se realizó con el antígeno de Huddleson, pero el portaobjetos se movió a mano o con agitador rotatorio durante solo tres minutos a $100 \mathrm{rpm}{ }^{(1)}$.

Se consideró reacción positiva en presencia de aglutinación débil o intensa, visible microscópicamente, mientras que la reacción negativa se evidencio una suspensión uniforme sin cambio visible alguno, tal como se presentó en el control negativo.

\section{Factores predisponentes}

Los factores predisponentes de brucelosis, se determinaron aplicando una encuesta validada por la Dirección General de Epidemiologia, en el año 2012, donde se recogieron datos como la ocupación, alimentación y contacto directo con productos de desecho, tejidos o excretas de animales enfermos o portadores asintomáticos, entre otros.

\section{Análisis de datos}

Por último el análisis estadístico de los datos se realizó con el paquete SPSS para Windows versión 19.0. Se emplearon las proporciones para los datos cualitativos y los promedios y DS (desviación estándar) para los cuantitativos con el fin de determinar la prevalencia de brucelosis y los factores predisponentes de la enfermedad.

\section{Consideraciones éticas}

Las personas que formaron parte de este estudio aceptaron voluntariamente, se les explicó detalladamente la razón por la que extrajo la muestra sanguínea y firmaron un consentimiento informado.

Las muestras y encuestas se identificaron usando una codificación numérica seriada. En todo momento se mantuvo la confidencialidad de la información y se usó solo con fines de la presente investigación.

\section{RESULTADOS}

Se evaluaron 131 personas de ambos géneros. Encontrándose que el $10,7 \%$ de los trabajadores resultaron seropositivos a las pruebas de Rosa de Bengala y Huddlenson, siendo el $15,4 \%$ del género femenino y el $10,2 \%$ del género masculino seropositivos a dichas pruebas.

Además se observó que la ocupación de matarife presentó la mayor frecuencia de bruceosis $(8,1 \%)$, representando el $64,3 \%(9 / 14)$ de los positivos (Tabla 1 ).

Tabla 1. Seroprevalencia de brucelosis en los trabajadores de mataderos de la región Lambayeque según la ocupación. Junio 2014 - Febrero 2015.

\begin{tabular}{lcccccc}
\hline Ocupación & \multicolumn{2}{c}{ Seronegativo } & \multicolumn{2}{c}{ Seropositivo } & \multicolumn{2}{c}{ Total } \\
\hline \multirow{2}{*}{ Administrador } & $\mathbf{N}$ & $\%$ & $\mathbf{N}$ & $\%$ & $\mathbf{N}$ & $\%$ \\
Limpieza & 3 & 75,0 & 1 & 25,0 & 4 & 100,0 \\
Matarife & 5 & 71,4 & 2 & 28,6 & 7 & 100,0 \\
Veterinario & 102 & 91,9 & 9 & 8,1 & 111 & 100,0 \\
Total General & 7 & 77,8 & 2 & 22,2 & 9 & 100,0 \\
\hline
\end{tabular}


Asimismo se observó que 13 de los trabajadores evaluados presentaron sintomatología, y de dichos trabajadores el $46,2 \%$ (6/13) presentaron anticuerpos anti - Brucella sp. También se observó que el 11,0\% (9/14) de trabajadores que presentaban heridas o cortes en la piel durante el sacrificio de los animales fueron seropositivos.

Respecto al riesgo de contaminación por consumo de sangre, fetos y placentas de animales enfermos, se observó que el $16,7 \%, 25,6 \%$ y $15,4 \%$ de los trabajadores eran seropositivos para brucelosis, respetivamente (Gráfico 1).

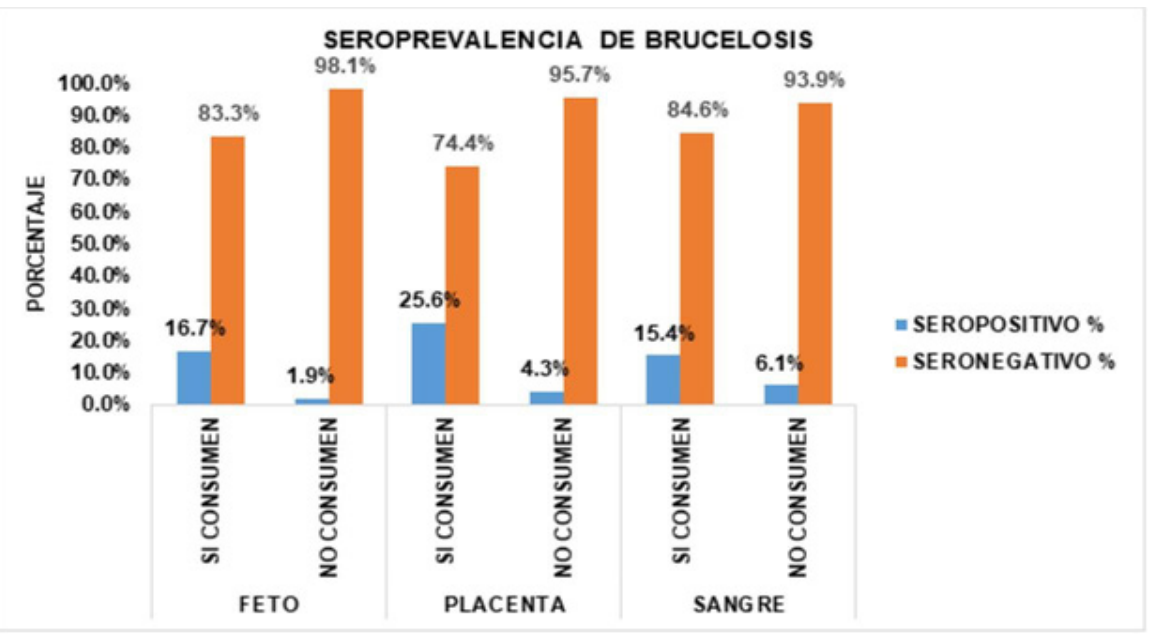

Gráfico 1. Seroprevalencia de brucelosis en los trabajadores de mataderos de la región Lambayeque según el consumo de fetos, placentas y sangre. junio 2014 - febrero 2015.

\section{DISCUSIÓN}

Se procesaron 131 muestras sanguíneas de personas de ambos géneros. Encontrándose una seroprevalencia de brucelosis del $10,7 \%$ en los trabajadores de mataderos de la región Lambayeque, resultado que no superó al reportado por un estudio previo, el cual fue del $14,0 \%$ en pacientes atendidos en un hospital local, encontrando dicho autor relación significativa en el género y la presencia de brucelosis (7), caso contrario en esta investigación no se pudo establecer esta relación.

Para determinar la seroprevalencia de brucelosis se realizaron las pruebas de Rosa de Bengala y Huddleson, mientras que en otra investigación donde se también trabajó con Rosa de Bengala y PCR se obtuvo $47,0 \%$ y $13,0 \%$ de seropositividad respectivamente.

Los valores obtenidos con Rosa de Bengala difieren de los obtenidos con PCR, lo que podría deberse a que la prueba de Rosa de Bengala no es método confirmatorio, a pesar de tener alta confiabilidad se trata de una prueba tamiz que solo informa sobre presencia de inmunoglobulinas $\mathrm{G}$ y $\mathrm{M}$ en el suero sanguíneo ${ }^{(17)}$, además ninguna prueba por sí sola es suficiente para establecer un diagnóstico, por lo que se emplearon dos pruebas serológicas con alta especificidad para darle mayor validez al estudio.

Diversos investigadores, trabajaron con brucelosis caprina utilizando también el antígeno Rosa de Bengala como en el presente estudio, debido a su alta efectividad y rapidez obteniendo buenos resultados ${ }^{(18,19)}$. Además concluyeron que existe relación entre la brucelosis caprina y humana,existiendo por cada persona enferma 69 cabras afectadas (20). Siendo la mayor dificultad en el control de la Brucelosis animal el sistema de crianza nómade y la eliminación de los reactores positivos, debido a la idiosincrasia de la mayoría de los criadores; lo que se ve reflejado en la prevalencia de brucelosis humana por su amplia relación (4).

La brucelosis presenta sintomatología dispersa, lo que muchas veces dificulta su diagnóstico, ya que los signos y síntomas suelen confundirse con los de otras patologías, incluso los pacientes muchas veces son asintomáticos en las primeras etapas de la enfermedad.

En el presente estudio se halló que el 10,7\% de los trabajadores tenían síntomas, de los cuales el $46,2 \%$ presentó anticuerpos anti Brucella sp, además el síntoma de mayor frecuencia fue el dolor de cabeza.

Mientras que, en un estudio similar fueron la fiebre intermitente, artralgias y debilidad los de mayor frecuencia (21). Sin embargo no se encontró relación entre la aparición de la enfermedad la sintomatología.

De acuerdo a la ocupación de los trabajadores, se encontró que el $28,6 \%$ del personal limpieza, 25,0\% personal administrativo, $22,2 \%$ veterinarios y $8,1 \%$ matarifes fueron seropositivos a Brucella sp (Tabla 2). Resultados similares a los hallados por Jugeshuarsingh y Orta, donde el 7,0\% de matarifes, $1,0 \%$ de personal de limpieza y $1,0 \%$ de veteri 
narios fueron seropositivos a Brucella sp. (22), mientras otro estudio halló que el $71,0 \%$ pacientes evaluados presentó infección brucelósica debido al ambiente laboral en el que se desempeñaban ${ }^{(23)}$.

En la investigación de López y colaboradores, se evaluaron trabajadores de una planta de residuos sanitarios, encontrando un $33,7 \%$ de infectados, haciendo hincapié en la importancia de riesgo ocupacional en la diseminación de la enfermedad ${ }^{(24)}$

Cabe recalcar que, la brucelosis se transmite al hombre principalmente por tres vías, mediante contacto directo con animales enfermos o sus productos, sea el caso de sangre, orina, descargas vaginales, fetos abortados o placentas de animales infectados; por inhalación de partículas que contengan al microorganismo y por la ingestión de alimentos contaminados como leche, quesos contaminados o carne mal cocida.

En la transmisión de brucelosis la transmisión por contacto directo juega un papel importante la presencia de heridas producidas durante la faena y que no hayan cicatrizado. En la tabla 4 se muestra que un total de 82 trabajadores si presentaron heridas no cicatrizadas durante el sacrificio, de los cuales el $11,0 \%$ fueron seropositivos a Brucella sp; sin embargo no se encontró relación directa entre la presencia de heridas no cicatrizadas durante el sacrificio de los animales y la infección con Brucella sp.

Respecto a las medidas de seguridad en el trabajo se encontró que el $26,7 \%$ no usó protección durante la faena. Resultados similares a los obtenidos por Cedeño y Navas25, donde el $38,0 \%$ de los trabajadores no emplearon protección durante la matanza. Así también Jugeshuarsingh y Orta 22 encontraron que solo el $18,0 \%$ usaba protección; lo que pone de manifiesto en todos los casos que es un gran porcentaje de trabajadores no usan medidas de protección durante el sacrificio de los animales, lo que podría deberse a que, según manifestaciones de los mismos, es incómodo, ya que se resbalan los utensilios, además que los trabajadores desconocen las medidas preventivas a seguir y que se les permite la realización de la faena en condiciones sanitarias inadecuadas.

Cabe resaltar que, el $83,5 \%$ de los mataderos visitados tenían condiciones higiénicas inadecuadas o deficientes para el sacrificio de los animales y considerando que la mala higiene, inadecuada disposición de las excretas, sangre, tejidos y fluidos de los animales, favorece la dispersión del microorganismo a través de areosoles, esta puede ser una vía de ingreso del microorganismo.

De acuerdo a la ingestión de alimentos contaminados se reporta que, $25,6 \%$ de los trabajadores consumía habitualmente placentas, $16,7 \%$ fetos y otro $15,4 \%$ sangre de ani males infectados con Brucella sp. (Grafica 1). En un estudio realizado en trabajadores de mataderos, el principal factor de riesgo fue el consumo de placentas $(87,6 \%)^{(25)}$, además reportaron que el $34,5 \%$ de los trabajadores consumió sangre infectada con el microorganismo ${ }^{(25)}$.

Así también Ron y colaboradores manifestaron que el consumo de fetos y placentas constituye un factor de riesgo importante para la aparición de la enfermedad (26), lo que podría deberse a la afinidad de $B$. abortus por el endometrio grávido y placenta fetal, por lo que este microorganismo prolifera en el tejido placentario que rodea al feto, su predilección por estos tejidos se atribuye a la presencia del i-eritritol, ya que constituye una fuente de energía para el crecimiento de Brucella sp ${ }^{(8)}$.

Cuando Brucella $s p$ ingresa al huésped por el tracto gastrointestinal, es fagocitada, y capaz de resistir los mecanismos de destrucción intracelular; dentro de los macrófagos se disemina por el organismo en el torrente sanguíneo y se multiplica en el sistema retículoendotelial de hígado, bazo, médula ósea, ganglios linfáticos y otros órganos. Se observó que el $15,4 \%$ los matarifes que consumían sangre presentaron anticuerpos anti Brucella $s p$, según la prueba de chi cuadrado $(p<0,05)$ se determinó que existe relación entre el consumo de sangre de animales sacrificados y la presencia de anticuerpos anti Brucella sp en los matarifes, y por lo tanto el principal factor predisponente fue el consumo de un producto contaminado, específicamente el consumo de la sangre.

En tanto diversos investigadores coincidieron también en que la principal forma de contagio de brucelosis es el contacto directo con animales y el consumo de productos contaminados con el microorganismo ${ }^{(6,10)}$.

Se resalta la importancia de promover sistemas de vigilancia epidemiológica para brucelosis en todos los mataderos de la región Lambayeque, además de fiscalizar a aquellos que operan de manera clandestina, ya que no se cuenta con información acerca de las condiciones del ganado sacrificado y de la utilización de medidas protección para los trabajadores.

Con la finalidad de detectar a tiempo factores que predispongan el inicio y desarrollo de esta y otras enfermedades. Además se sugiere la capacitación de los trabajadores, dándoles a conocer cuáles son medidas sanitarias y los elementos de protección necesarios para evitar la transmisión de Brucella sp., concientizándolos sobre la importancia de su utilización durante sus actividades, evitando de esta manera, la contaminación de la piel, inhalación o ingestión accidental del microorganismo.

En conclusión, la seroprevalencia de brucelosis en personal que labora en mataderos de la región Lambayeque, durante los meses de junio 2014 a febrero 2015 , fue mayor a $10 \%$, 
revelando la importancia de esta enfermedad en población ocupacional de riesgo.

Asimismo el factor predisponente de mayor importancia para la transmisión de brucelosis en los trabajadores fue el consumo de productos de animales infectados con Brucella sp., específicamente el consumo de sangre de animales sacrificados.

\section{Fuentes de financiamiento. Autofinanciado.}

\section{Conflicto de interés: Ninguno.}

Agradecimiento. A la Universidad Nacional Pedro Ruiz Gallo, que brindó el ambiente adecuado y equipo requerido para el procesamiento de las muestras biológicas.

\section{REFERENCIAS BIBLIOGRAFICAS}

1. Organización Mundial de la Salud. Zoonosis y Enfermedades Transmisibles Comunes al Hombre y a los Animales. Rev. OMS. 2003; 1: 28-40.

2. Oficina Internacional de Epizootias-OIE. Brucelosis Bovina: Manual de Normas para Pruebas de Diagnóstico y Vacunas. Rev. OIE. 2004; 3: 2856.

3. Sbriglio H y Saenz S. Brucelosis. Rev. Bioanálisis. 2007; 1: 18-22.

4. Navarro A, Bustamante J y Guillen A. Estrategias de prevención y control de la brucelosis humana en el Perú. Rev Perú Med Exp Salud Pública. 2005; 22(2): 87.

5. Instituto Nacional de Salud. Brucelosis: Boletín informativo epidemiológico. Instituto Nacional de Salud. 2014; 56: 23-24.

6. Vélez M. Diagnóstico serológico de brucelosis caprina en la provincia de Lambayeque. [Tesis para optar por el título profesional de Médico veterinario]. Facultad Medicina Veterinaria de la Universidad Nacional Pedro Ruiz Gallo; 1991.

7. Alamas J. Factores predisponentes y diagnóstico serológico de Brucelosis en personas atendidas en el hospital docente "Belén". Provincia de Lambayeque. Abril 2001- Marzo 2002. [Tesis para optar por el título profesional de Biólogo - Microbiólogo y Parasitólogo]. Facultad de Biología de la Universidad Nacional Pedro Ruiz Gallo; 2002.

8 Castro H, Gonzáles S y Prat M. Brucelosis: una revisión práctica. Acta Bioquim. Clín. Latinoam. 2005; 39(2): 203-216.

9. López A. Brucelosis: Nuevos retos en el control. Brucelosis, un problema de salud pública aún sin resolver. XVIII Congreso Panamericano de Ciencias Veterinarias. La Habana: PANVET. 2002.

10.Jozic B. y Mosquera O. Prevalencia de brucelosis humana en personal de riesgo y brucelosis bovina en la sala de matanza Ticoporo del Municipio Antonio José De Sucre, Barinas, Venezuela, durante el primer semestre del 2004. Gac. Cs. Vet. 2004; 8(2): 69-78.

11.Lugo A, Pérez M, Hernández Y y Rosales D. Brucelosis humana en estudiantes de la escuela de bioanálisis, Universidad de los Andes-Venezuela. Rev. Salud Publ. y Nutric. 2010; 11(4): 20-23.

12.Ministerio de Salud del Perú (MINSA). Manual de procedimientos de laboratorio en técnicas básicas de hematología. Ministerio de salud. 2005a; 1: $14-19$.

13.Acha PN y Szyfres B. Zoonosis y enfermedades transmisibles comunes al hombre y a los animales. 3a ed. Washington: Edit. Organización Panamericana de la Salud. 2001. 398p.

14. Herrera S y Cárdenas M. Brucelosis: Diagnóstico serológico y vacunas. Instituto Nacional de Salud. 2003. 45p.

15. Milton S y Tsokos T. Estadística para biología y ciencias de la salud. $1^{\mathrm{a}} \mathrm{ed}$. Madrid: Edit. Mc Graw-Hill-Interamericana; 2001.

16. Ministerio de Salud del Perú (MINSA). Norma técnica de diagnóstico y tratamiento de brucelosis humana. Ministerio de salud. 2005b; 1: $9-13$.

17.Cevallos O, Carranza M, Saucedo S, Romero D, Ramos L y Reyes X. Diagnóstico serológico (Rosa de Bengala) y molecular (PCR) de brucelosis en humano. Rev. Ciencia y tecnología. 2010; 1(3):27-32.

18.Garro E, Delgado A, Evaristo R y Manchego A. Prevalencia de Brucelosis caprina en la provincia de Barranca, Lima. Rev. Inv. Vet. 2005; 16 (2): $184-6$.
19. Toledo E, Delgado C, Suarez A y Noé M. Prevalencia de Brucelosis Caprina en tres distritos de la provincia de Cañete, Lima. Rev. Inv. Vet. Perú. 2007; 18(2): 136-40.

20. Herrera DI, Abeledo A, Rodríguez MA, Jacome JA, Rivera EL y Vallecillo JA. Prevalencia de Brucelosis caprina y su relación con la humana en Tenextepec, Municipio de Perote, Veracruz, México. Rev. Salud Anim. 2001; 23(3): 160-4.

21.Reyes J, Sánchez M, Lotero M, Restrepo M y Palacio L. Seroprevalencia e incidencia de Brucella sp en vacunadores del Programa para el contro de brucelosis bovina, en el Departamento de Antioquia-Colombia. Rev. Colombiana de Ciencias Pecuarias. 2010; 23(1): 35-46.

22.Jugeshuarsingh AL y Orta AR. Seroprevalencia de Brucella abortus en los trabajadores de mataderos. Estado Monagas. [Tesis para optar por el título profesional de Médico cirujano]. Facultad de Medicina de la Universidad de Oriente Núcleo Bolívar; 2010.

23. Serra AJ y Godoy GP. Incidencia, etiología y epidemiología de la brucelosis en un área rural de la provincia de Lleida. Rev. Esp. Salud y Vida. 2000; 74(1): 45-53

24.López Hernández B, Almagro Nievas D y Cabrera Castillo MJ. Seroprevalencia de brucelosis en los trabajadores de una planta de tratamiento de residuos biosanitarios. Med. Clin. 2003; 120(10): 376-7.

25.Cedeño MA y Navas CA. Prevalencia de brucelosis en faenadores, veterinarios y administradores de los camales en la provincia de Manabí, mediante prueba Rosa de Bengala y suero aglutinación en tubo. [Tesis para optar por el título profesional de Médico Veterinario]. Ecuador: Facultad de Medicina Veterinaria, Universidad Tec. de Manabí; 2007.

26. Ron J, Abatih E, Celi M, Vizcaíno L, Calva J y González P. Human brucellosis in northwest Ecuador: typifying Brucella spp., seroprevalence, and associated risk factors. Rev. Vector Borne Zoonotic Dis. 2014; 14(2): $124-$ 33

Revisión de pares: Recibido: 03/02//2019 Aceptado: 01/04/2019 